\title{
THE ROLE OF SPINAL CORD FACILITATION IN NEUROGENIC BLADDER FUNCTION
}

\author{
By Professor Arthur S. Abramson, M.D., and Assoc. Professor Matei S. Roussan, M.D. \\ Department of Rehabilitation Medicine, Albert Einstein College of Medicine, \\ Bronx, New York I046I
}

THE subject of this paper is the individual with neurogenic bladder resulting from traumatic transverse lesion of the spinal cord. For this kind of patient the goals of management are well established. The urine should be kept sterile, the bladder should remain of modest capacity and free of ureterovesical reflux and stone, it should be possible to empty it easily and completely within a reasonably short period of time following the injury and autonomic phenomena resulting from bladder filling should remain minimal. With more advanced methods of management the patient is more likely to reach these goals. However, with more traditional methods, these goals are much harder to achieve. The indwelling catheter acts as a constant irritant; frank urinary infection or at least bacteriuria are almost inevitable, the incidences of reflux and stone are high; satisfactory bladder emptying is much less sure and autonomic phenomena can be severe. This is the sequence of events frequently seen in the individual with spinal cord injury especially when the lesion is at a high level. In addition, other complications, such as decubiti, may occur with inadequate care. In such cases, establishment of effective voiding is difficult. The most important reason for this difficulty is the tendency of the spinal cord to facilitate.

Facilitation of the cord depends on a number of factors such as the nature of the central excitatory state as well as temporal and spatial summation of afferent impulses.

\section{THE CENTRAL EXCITATORY STATE}

The central excitatory state determines the ultimately stabilised level of spacticity after diaschisis has passed off. Its heightened level is due to disinhibition of the isolated cord. Its nature depends upon the kind of stimulation and quantity of impulses arriving at the cord during its functional reorganisation as the patient slowly changes from a flaccid to a spastic state. In other words, the individual who is beset with urinary infection, stone, indwelling catheter and decubiti during the early period after cord injury affords rich sources of noxious stimulation and continuous afferent flow of impulses. Such an individual is more likely to develop severe spasticity than is one who has been relatively free of complications. When the stabilised level of spasticity is severe, it reflects a very hyperactive central excitatory state highly responsive to temporal and spatial summation, a responsiveness manifested in both the somatic and autonomic nervous systems.

While the central excitatory state is a fairly stable phenomenon after it becomes well established, it can be altered when fresh sources of noxious stimulation appear. An example of this is the individual who develops decubiti or bladder stone after stabilisation has occurred. The most usual result is markedly increased 
activity of the somatic and autonomic nervous systems during bladder filling. With healing of the decubiti or the removal of the stone the activity is often greatly reduced.

\section{TEMPORAL SUMMATION}

Increased reflex responsiveness occurs when afferent impulses reach the cord in rapid succession. This is called temporal summation. The shorter the interval between impulses, the greater the responsiveness. Stimulation afforded by bladder action is such a source of temporal summation. The bladder can fill without great increase in intracystic pressure but not without marked increase in wall tension. Iggo (1955) has produced strong indirect evidence that the stretch endorgans in mouse bladder wall are in series with contractile elements of the detrusor muscle. This has a special significance in that these end-organs should be responsive not only to stretching of the bladder wall but also to contraction of the stretched wall. Iggo has also shown that increasing wall tension produces a rapid succession of afferent impulses with continuously shortening intervals between impulses. Thus, the bladder, as it fills, is unique in that it does not produce a constant form of stimulation permitting accommodation to occur, but rather a constantly changing form of stimulation resulting in a constantly increasing responsiveness of the spinal cord.

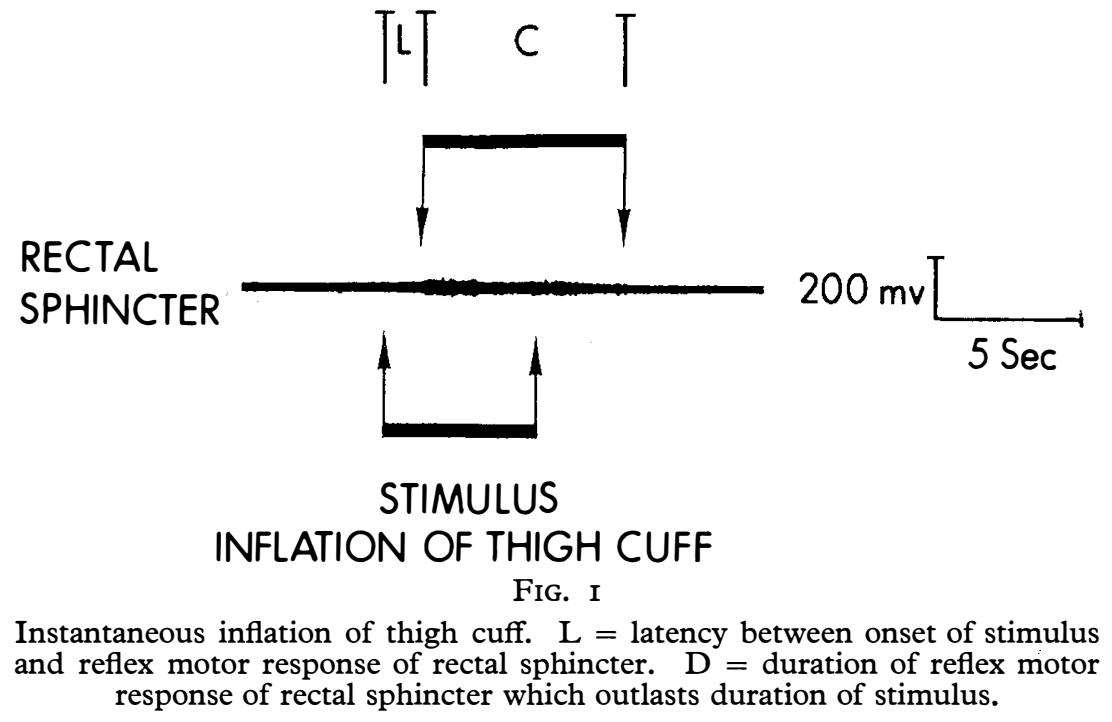

\section{SPATIAL SUMMATION}

When responsiveness is enhanced by stimuli being applied simultaneously or in rapid succession to two or more separate afferent nerves, the process is called spatial summation. This effect of mutual support and reinforcement of allied reflexes constitutes the form of facilitation most commonly seen with bladder filling. 
The phenomenon of facilitation can be demonstrated in the human by a simple clinical experiment. In an individual with a high transverse lesion of the spinal cord and with a hyperactive central excitatory state, the temporal summation

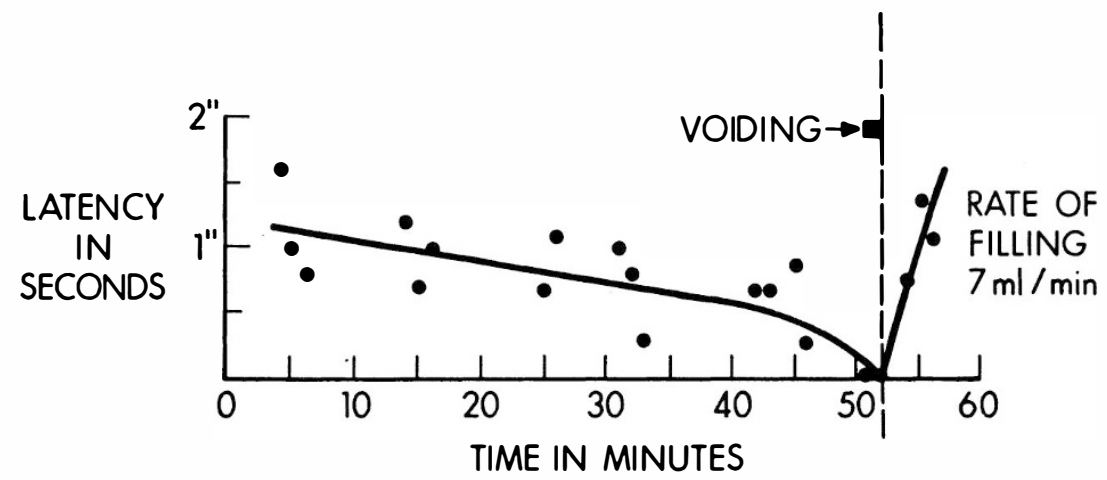

FIG. 2

Relationship of duration of latency and bladder filling. Latency shortens as bladder fills and lengthens with emptying.

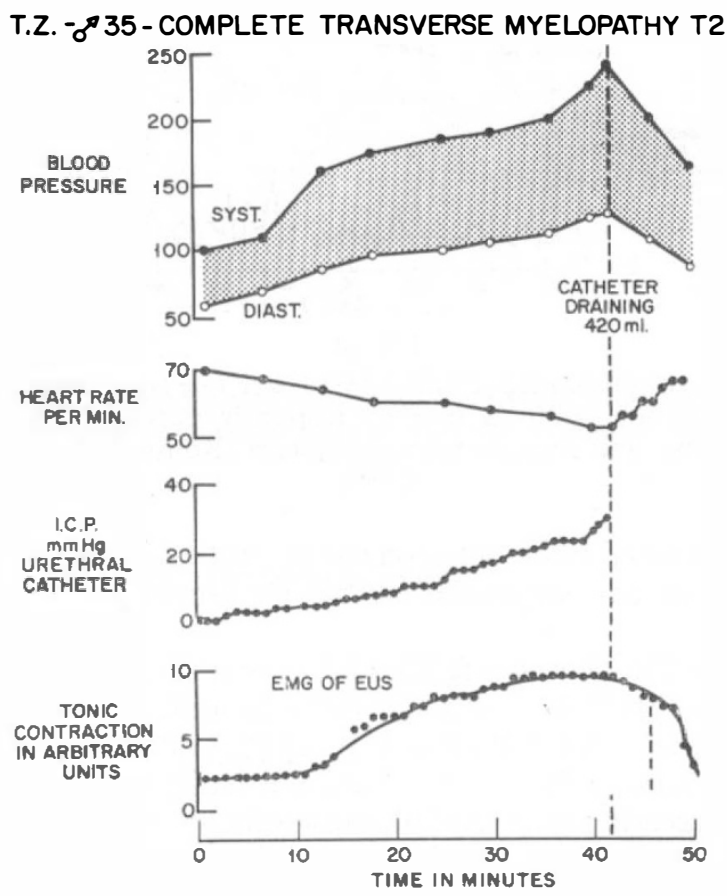

FIG. 3

Typical response to bladder filling in patient with high complete transverse lesion of spinal cord gradually increasing hypertension, bradycardia, bladder and sphincter tone (Roussan et al., I966). 
resulting from bladder filling continuously increases the tendency to facilitation. A fixed stimulus periodically applied to a reflexogenic zone elsewhere below the level of the lesion produces a contractile response of the rectal sphincter (fig. I). The latency between stimulus and response continuously shortens as the bladder is continuously being filled (fig. 2). Emptying of the filled bladder rapidly restores the resting latency. The duration of the motor response lengthens as the latency shortens and here too, the resting duration is restored with emptying. This

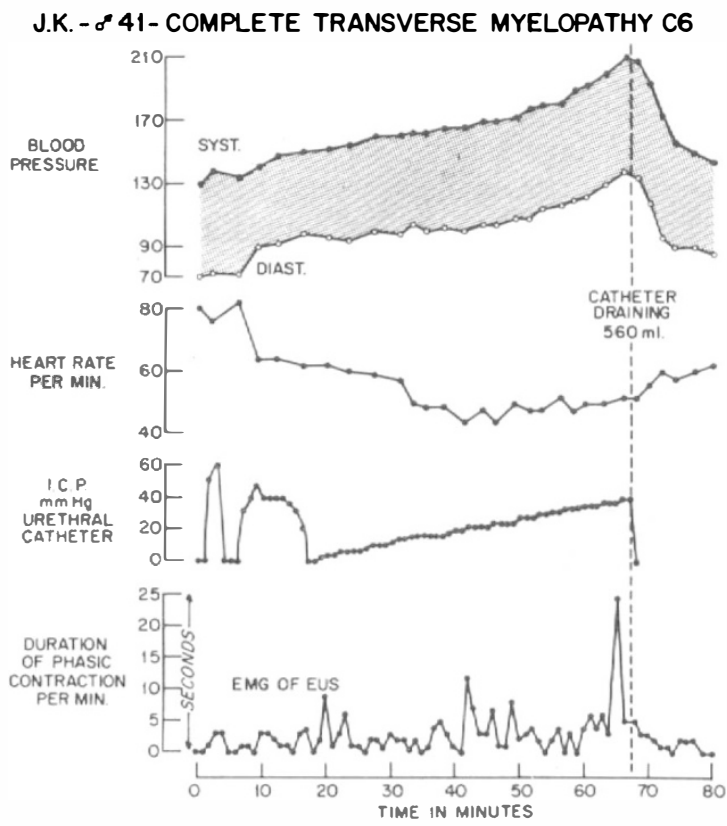

FIG. 4

Early appearance of uninhibited detrusor contraction does not stimulate marked responsiveness of autonomic and somatic nervous system (Roussan et al., 1966).

demonstration of the interaction between the central excitatory state, temporal and spatial summation can be illuminated further by functional studies of the neurogenic bladder.

The function of the neurogenic bladder is best studied by simultaneously recording significant events in both somatic and autonomic nervous systems throughout bladder filling (Guttmann \& Whitteridge, 1947; Guttman, 1954; Roussan et al., I966). The basic events to be observed are a steadily rising blood pressure and falling heart rate, increasing tonic and phasic sphincter contractions and a slow but steady elevation of intracystic pressure (fig. 3). The nature of the central excitatory state determines the rapidity and extent of these changes. This type of rise of intracystic pressure is never very marked. It is analogous to the increasing tone of the sphincter. Unpredictably, short-lasting phasic contractions of the detrusor muscle can be superimposed on this rising baseline. These have been called uninhibited detrusor contractions. When they occur early, the reflex effects of 


\section{ES. ó 39: Transverse Myelopalhy, T3, Conplete.}

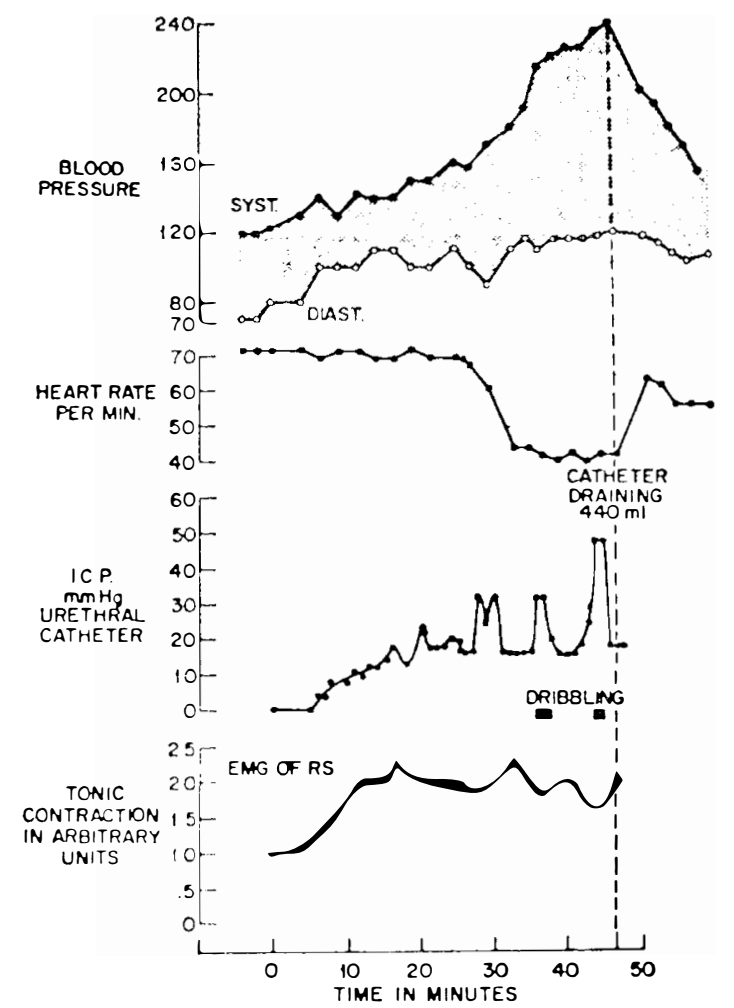

Fig. 5

Late appearance of uninhibited detrusor contractions stimulates marked responsiveness (Roussan et al., I966).

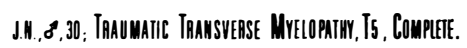

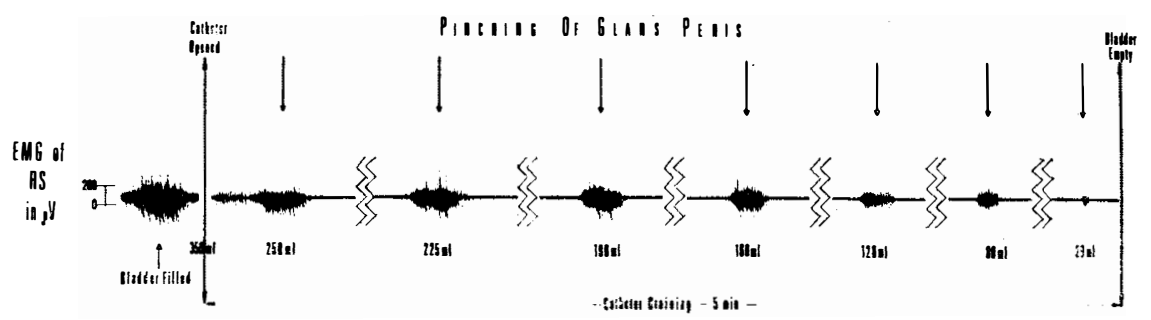

FIG. 6

Spincter contraction in response to a stimulus progressively decreases as the bladder is gradually emptied. 
such phasic contractions on the other events being recorded are negligible (fig. 4). When they occur late, the effects can be quite marked (fig. 5). Simply stated, this means that increasing facilitation occurs throughout bladder filling which is further enhanced by contraction of the filled bladder. This indirect clinical evidence for a serial arrangement of stretch end-organs in the human is suggestive. If that is the arrangement and facilitation the result, doubt must be cast upon the assumption that simple emptying will occur upon artifically stimulating the filled bladder. Instead, more intense sphincter contraction and autonomic phenomena should be expected.

Further evidence for the pervasive influence of spinal cord facilitation is provided by observing the phenomenon of defacilitation. If reflex sphincter contraction is observed to increase as the bladder fills it should also be observed to decrease as the bladder empties. Such is the case. A suitable patient was drained of $350 \mathrm{cc}$. of bladder content over a period of 5 minutes. The reflex sphincter response to periodic pinching of the glans progressively decreased as the volume within the bladder lessened (fig. 6).

\section{COMMENT}

Facilitation is not limited to, but merely exaggerated in the abnormal state. It occurs in the normal where its presence is heralded by a mild elevation of blood pressure during the emptying contraction. It is a variable phenomenon since its severity is not determined entirely by the nature of the cord lesion. Note has already been made of the clinical impression that it is more severe in those more beset with early complications. Evidence for this impression is contained in the early report of Head and Riddoch (1917) who described extraordinarily severe spasticity in the terribly complicated patients of 50 years previous. Such severe clinical states are rarely seen with the more skilful clinical management of modern times. A regimen designed to prevent complications totally would be a major step forward in controlling facilitation, at least partially. Nevertheless, it still remains a troublesome problem in many patients. It more or less causes obstruction to urine outflow because of heightening sphincter activity during filling and because the sphincter has difficulty relaxing when the bladder is full. This is the opposite effect as compared with the reciprocal action between the smooth muscle detrusor and the striate muscle sphincter as is seen in the normal. When sphincter activity is not too excessive some urine flow can occur, especially when assisted but, too often, residual urine remains. When sphincter activity is excessive urine flow is largely interdicted and autonomic phenomena tend to be severe.

The idea of the bladder as a contractile bag which could be emptied by direct artificial stimulation is oversimplistic. Earlier workers in this field approached the problem without giving sufficient consideration to the neurophysiologic principles which were involved and their results were less than adequate (Lancet, 1972). Occasional cases of complete emptying have been reported but usually by those who attempted to fatigue, denervate or cut the sphincter. At least, this indicates that increasing attention is being paid to sphincter activity as part of this very complex process. It is interesting to note that one investigator remarked on an 'unexpectedly' high blood pressure accompanying stimulation. He should have 'expected' such a result. The lesson to be learned is that in any bio-engineering system, the engineering component must be subservient to the biological problem and not vice versa. 
A pharmacological approach with the intent of suppressing the tendency of the spinal cord to facilitate seems to be more promising. There are some agents which can, on occasion, afford quite good results. An example of such an agent is CIBA Ba-34647 (Liorésal) a gamma aminobutyric acid derivative (Jones et al., I970). In a pilot study of three cases of severe facilitation, it markedly reduced sphincter activity permitting spontaneous and relatively complete emptying of the bladder in one case and the opportunity to empty with assistance in the other two. There were also smaller blood pressure elevations. While it is still too early to predict the value of its universal use, it is nevertheless, an example of the possibilities inherent in the pharmacological approach as an answer to the problem of facilitation.

\section{SUMMARY}

Facilitation is defined as the biasing of the cord by the passage of a train of afferent impulses so that the reflex effect of a simultaneously or consecutively applied stimulus is enhanced. The tendency to facilitation is increased as a result of cord transection.

The urinary bladder is unique in that during filling, afferent impulses produced by stretching of the detrusor constantly increase in frequency. As a result, accommodation, as to a steady stimulus, does not occur. The effects are widespread in the somatic and autonomic nervous systems. Voiding may be prevented by excessive sphincter contraction and excessive autonomic phenomena may occur. Facilitation thus leads to the obliteration of reciprocal action between bladder and sphincter upon which voiding depends.

Artificial stimulation of the filled bladder further enhances facilitation and this may explain the shortcomings of such procedures.

The most logical approach to neurogenic bladder management is to suppress the tendency of the spinal cord to facilitate, by pharmacological means, if possible.

\section{REFERENCES}

Guttmann, L. \& Whitteridge, D. (1947). Effects of bladder distension on autonomic mechanisms after spinal cord injuries. Brain, 70, 36I-405.

GuttmanN, L. (1954). Visceral activity and peripheral circulation, London: T. \& E. Churchill.

HEAD, H. \& RIDDOCH, G. (I9I7). The automatic bladder, excessive sweating and some other reflex conditions in gross injuries of the spinal cord. Brain, 40, 188-263.

IGgo, A. (1955). Tension receptors in the stomach and the urinary bladder. F. Physiol. I28, 593-607.

Jones, R. F., Burke, D., Marosszeky, J. E. \& Gillies, J. D. (I970). A new agent for the control of spasticity. F. Neurol. Neurosurg, Psychiat. 33, 464-468.

Lancet Editorial (1972). Electrical stimulation of the bladder. I, 887.

Roussan, M. S., Abramson, A. S., Lippmann, H. I. \& D'Oronzio, G. (I966). Somatic and autonomic responses to bladder filling in patients with complete transverse myelopathy. Arch. Phys. Med. Rehab. 47, 450-456.

Thompson, C. E. \& Witham, A. C. (I948). Paroxysmal hypertension in spinal cord injuries. New England F. Med. 239, 29 I. 